\title{
Improving the Gene Transfection in Human Embryonic Stem Cells: Balancing with Cytotoxicity and Pluripotent Maintenance
}

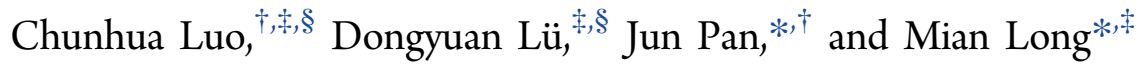

${ }^{\dagger}$ Key Laboratory for Biorheological Science and Technology of Ministry of Education, College of Bioengineering, Chongqing University, Chongqing 400044, China

${ }^{\ddagger}$ Center for Biomechanics and Bioengineering, Key Laboratory of Microgravity (National Microgravity Laboratory) and Beijing Key Laboratory of Engineered Construction and Mechanobiology, Institute of Mechanics, Chinese Academy of Sciences, Beijing 100190, China

ABSTRACT: Manipulation of genes in human embryonic stem cells (hESCs) is imperative for their highly potential applications; however, the transduction efficiency remains very low. Although existing evidence revealed the type, size, and zeta potential of vector affect gene transfection efficiency in cells, the systematic study in hESCs is scarce. In this study, using poly(amidoamine) (PAMAM) dendrimers ended with amine, hydroxyl, or carboxyl as model, we tested the influences of size and surface group as well as cytotoxicity and endocytosis on hESC gene transfection. We found that in culture

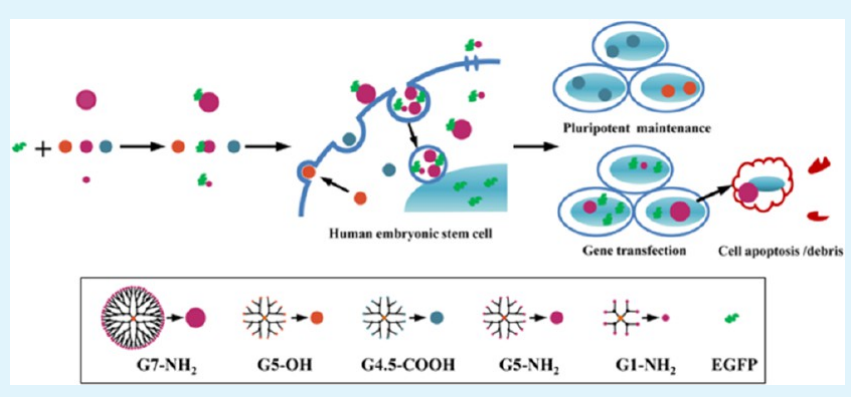
medium of mTeSR the particle sizes of G5, G7, G4.5COOH, and $\mathrm{G} 5 \mathrm{OH}$ were around $5 \mathrm{~nm}$ and G1 had a smaller size of $3.14 \mathrm{~nm}$. G5 and G7 had a slight and significant positive zeta potential, respectively, whereas G1 was slightly negative, and $\mathrm{G} 4.5 \mathrm{COOH}$ and $\mathrm{G} 5 \mathrm{OH}$ were significantly negative. We demonstrated that only amine-terminated dendrimers accomplished gene transfection in hESCs, which is greater than that from Lipofectamine 2000 transfection. Ten micromolar G5 had the greatest efficiency and was better than $1000 \mu$ M G1. Only a low concentration $(0.5$ and $1 \mu \mathrm{M})$ of G7 realized gene delivery. Amine-ended dendrimers, especially with higher generations, were detrimental to the growth and pluripotent maintenance of hESCs. In contrast, similarly sized hydroxyl- and carboxyl-terminated dendrimers exerted much lower cytotoxicity, in which carboxyl-terminated dendrimer maintained pluripotency of hESCs. We also confirmed the endocytosis into and significant exocytosis from hESCs using FITC-labeled G5 dendrimer. These results suggested that careful considerations of size, concentration, and zeta potential, particularly the identity and position of groups, as well as minimized exocytosis in the design of a vector for hESC gene delivery are necessary, which helps to better design an effective vector in hESC gene transduction.

KEYWORDS: dendrimer, embryonic stem cells, gene transfection, end group, size, endocytosis

\section{INTRODUCTION}

Human embryonic stem cells (hESCs) are defined by their capacity to self-renew while maintaining their ability to differentiate into virtually any cell type. Due to their efficient clonal propagation, they have wide-reaching potential applications, such as cell replacement therapies and regenerative medicine. hESCs also serve as excellent models for the study of human embryonic development, organogenesis, pathology, and drug/biomaterial toxicity screening. In particular, hESCs are an unparalleled tool for manipulating the human genome. ${ }^{1,2}$ To realize gene regulation, exogenous genes should be transferred into hESCs. Upon successful gene delivery, stemness maintenance or directed differentiation of hESCs could be achieved. Furthermore, unknown genes could be discovered and their function could be studied and utilized in gene therapy and gene immunization, ${ }^{3}$ a possible strategy for treating various human disorders because most of these disorders are related to the genetic makeup of the patient. ${ }^{3,4}$ For example, the finding of ESC cycle-specific miRNAs reveals an important pluripotency regulation mechanism, where ESC-enriched or ESC-depleted miRNAs regulate the induction of pluripotent stem cells (iPSCs). ${ }^{3}$

A delivery system is required to protect, compact, and deliver genes expected to enter hESCs, since free genes are normally degraded in the systemic circulation and in vitro culture system. To date, many techniques have been used to transfer genes into hECSs to generate genetically stable modified hESC lines, including physical (electroporation), viral-mediated (infection), and chemical-based (transfection) procedures. Electroporation gives a poor efficiency of $\sim 2 \%$ in hESCs due to the low survival rates. ${ }^{5,6}$ Gene manipulation by viral infection produces a high percentage of modified cells. The most efficient lentiviral vectors with risk of insertional mutagenesis yield 22-25\% transduction efficiency in $\mathrm{H} 1$ and H9 hESCs. ${ }^{5}$ By removal of the feeder cells

Received: January 11, 2016

Accepted: March 14, 2016

Published: March 14, 2016 
and lowering the density of hESCs in a monolayer, the transduction efficiency is significantly improved. ${ }^{7}$ However, the safety concerns limit the use of viral vectors for human gene transfer, ${ }^{8}$ even though low-risk insertional mutagenesis vectors derived from adenovirus and adeno-associated virus (AAV) exist but have an unsatisfactory transduction efficiency of $\sim 11 \%$. ${ }^{9}$ Transfection is the most possibly used method for introducing transgenes into hESCs. It is straightforward, relatively easy to calibrate, provides a sufficient number of cells for clonal expansion, can be performed on adherent cell cultures, and allows the insertion of constructs of virtually unlimited size. ${ }^{5}$ A cationic star copolymer with a $\beta$-cyclodextrin core comprised of poly(2-dimethyl aminoethyl methacrylate) as the cationic component and poly(2-hydroxyethyl methacrylate) as the nontoxic stealth component reveals low toxicity and comparable or better transfection capabilities than Lipofectamine $2000{ }^{4}$ A cationic, hydrolytically degradable poly(beta-amino esters) presents an efficiency of $22 \%$, which is 4 -fold higher than that from Lipofectamine 2000 and maintains hESCs in undifferentiated state. ${ }^{10} \mathrm{~A}$ recent work reports a $50 \%$ transduction efficiency using a cell-binding ligand (RVG) conjugated redoxsensitive biodegradable dendrimer-type arginine-grafted polymer (PAM-ABP). ${ }^{11}$

Gene transduction in hESCs reveals very low efficiency in comparison to other cell types, especially the immortalized human tumor cell lines with almost complete efficiency. ${ }^{12}$ Generally, the delivery system is uptaken in cells through endocytosis or fusion of lipid vesicles to the plasma membrane. hESCs lack substantial phagocytic capacity. ${ }^{13}$ To be worse, hESCs are found to have profuse excretion of the gene delivery vectors. $^{14-17}$ To accelerate the hESC application, safe and efficient nonviral delivery system for hESC gene delivery is needed.

Cationic nanoparticles are promising candidates for hESC transduction, as has been shown in recent works. ${ }^{4,10,11}$ Cationic nanoparticles have amine end groups, which can integrate genes and cell membranes through charge interactions and promote endocytosis. However, previous studies have reported that the cationic nanoparticles, especially those larger in size, readily damage cell membranes and lead to cell lysis. ${ }^{18}$ On the other hand, small modifications of cationic polymer forming gene delivery nanoparticles, including modifications in size (in the range of $200-400 \mathrm{~nm}$ ) and zeta potential (in the range of $5-16 \mathrm{mV})$, greatly affected the gene transfection efficacy. ${ }^{10}$ Thus, it is essential to carefully design the cationic nanoparticles used for difficult-to-transfect hESCs, which are expected to not only efficiently deliver genes but also maintain viable and pluripotent (except of those for directing differentiation) hESCs.

Here the influences of size and surface group as well as cytotoxicity and endocytosis on the hESC gene transfection were tested systematically using poly(amidoamine) (PAMAM) dendrimers as model cationic nanoparticles. PAMAM dendrimers are tree-like molecules that grow from a central core and are composed of several layers (so-called generations). The reasons for choosing PAMAM are as follows. (1) They have widespread popularity in the gene delivery field and achieve gene delivery in hESCs. ${ }^{18}$ The positive charge at a physiological $\mathrm{pH}$ and "proton-sponge" effect from their secondary and tertiary amines in the core allow PAMAM to compact genes. The interaction of PAMAM with cellular membranes facilitates formation of small (15-40 nm), transient pores and entrance of PAMAM/ genes complex. ${ }^{19}$ (2) They have homogeneous molecular structure, globular architecture, nanometric size, and wide molecular weight range among different generations. (3) They have changeable surface functional groups besides amine, such as hydroxyl or carboxyl groups or even the arginine-glycine-aspartic acid (Arg-Gly-Asp, RGD) sequence. ${ }^{20}$

\section{EXPERIMENTAL SECTION}

Cell Culture. Human embryonic stem cells (H1) were grown on a matrigel-coated surface, added with $\mathrm{mTeSR}$ medium (Stem Cell Technologies, Vancouver, BC, Canada) at $37^{\circ} \mathrm{C}$ under a $5 \% \mathrm{CO}_{2}$ atmosphere. The culture medium was exchanged daily. Eight min Dispase (Stem Cell Technologies, Vancouver, BC, Canada) digestion was used for routine passage of hESCs. Undifferentiated colonies were carefully collected using a StemProEZ Passage cell scraper (Invitrogen, Waltham, MA) followed by gently pipetting into small aggregates, which were then dispensed into a fresh culture dish.

Dendrimer Characterization and Preparation. PAMAM dendrimers of amine-terminated generations one, five, and seven (G1, G5, and G7), carbonyl-terminated generation four and one-half $(\mathrm{G} 4.5 \mathrm{COOH})$, and hydroxyl-terminated generation five $(\mathrm{G} 5 \mathrm{OH})$ (Chengyuan Dendrimer Materials, Weihai, Shandong, China) were used in this study. G1, G5, and G7 were employed to assess the influence of particle size on gene transfection. By contrast, G5, G4.5COOH, and $\mathrm{G} 5 \mathrm{OH}$ were used to evaluate the impact of the end group on gene delivery upon previous observation that G5 is the best size for gene transduction for mesenchymal stem cells. ${ }^{20}$ Dendrimer particle sizes and zeta potential were measured by dynamic light scattering (DLS) and electrophoretic light scattering (ELS) using a Zetasizer Nano-ZS, Malvern Instruments (Worcestershire, UK), in water or mTeSR medium at a concentration of $100 \mu \mathrm{M}$ and $\mathrm{pH}$ 7.4. Dendrimers used for cellular study were dissolved in $\mathrm{mTeSR}$ medium at predetermined concentrations.

Enhanced Green Fluorescent Protein (EGFP) Transfection Using Dendrimer. Transfections were carried out using G1, G5, G7, $\mathrm{G} 4.5 \mathrm{COOH}$, or $\mathrm{G} 5 \mathrm{OH}$ as vector. The commercial Lipofectamine 2000 Reagent (Invitrogen, Waltham, MA) and EGFP plasmid (gift from Dr. Hucheng Zhao at the School of Aerospace Engineering, Tsinghua University) alone were used as controls. Different concentrations of dendrimers dissolved in $\mathrm{mTeSR}$ solution were incubated with $5 \mu \mathrm{g}$ of EGFP plasmid at room temperature for $1 \mathrm{~h}$. For transfection with Lipofectamine 2000, $5 \mu \mathrm{L}$ of Lipofectamine 2000 reaction solution and $5 \mu \mathrm{g}$ of EGFP plasmid were respectively diluted in $100 \mu \mathrm{L}$ of Opti-MEM medium (Invitrogen, Waltham, MA) at room temperature for $5 \mathrm{~min}$ followed by mixing and incubating at room temperature for $5 \mathrm{~min}$. The vector/plasmid complex or empty plasmid was added into the 2-day cultured hESCs and incubated for $6 \mathrm{~h}$ before being replaced with fresh mTeSR daily. The EGFP fluorescence intensity was monitored by confocal laser scanning microscopy (Zeiss L710, Jena, Germany) daily for 4 days. GFP-positive cells were counted and normalized to those from Lipofectamine 2000. Average green fluorescence intensity per cell at $96 \mathrm{~h}$ (since the fluorescence is brightest at that time point) was analyzed using ImageJ software (Bethesda, MD).

Cytotoxicity of Dendrimer in hESCs. hESCs seeded in 96-well plates at a density of 5-10 cell clones per well were allowed to attach overnight at $37{ }^{\circ} \mathrm{C}$ in a $5 \% \mathrm{CO}_{2}$ humidified incubator before being replaced with fresh $\mathrm{mTeSR}$ medium containing dendrimers in a concentration of $1,10,50$, or $100 \mu \mathrm{M}$. Dendrimer-containing medium was exchanged daily for 4 days. To test the time-dependent cytotoxicity, the cells cultured at a given day were incubated with CCK8 reaction solution (Beyotime, Nantong, Jiangsu, China) for $3 \mathrm{~h}$. The absorbance (OD) was read at $450 \mathrm{~nm}$ and then calculated as a fold related to the control (absence of dendrimer) or to the OD value on the first day.

Endocytosis of Dendrimer in hESCs. Endocytosis of G5 in hESCs was observed using confocal microscopy. G5 dendrimer was labeled with FITC (Sigma-Aldrich, St. Louis, MO) in water solution at a concentration of $0.36 \mathrm{~g} / \mathrm{mL}$ first. ${ }^{21}$ For this, FITC was dissolved in DMSO at a concentration of $1 \mathrm{mg} / \mathrm{mL}$ before being added to G5 solution, where the molar ratio of dendrimer to FITC was 1:50. The mixture was stirred overnight at room temperature followed by centrifugation three times with Fluorescent Dye Removal Columns 
Table 1. Mean Hydrodynamic Diameters and Zeta Potential of Dendrimers in Water or mTeSR Medium

\begin{tabular}{lrcrrr} 
& \multicolumn{2}{c}{ particle size $(\mathrm{nm})$} & & \multicolumn{2}{c}{ zeta potential $(\mathrm{mV})$} \\
\cline { 2 - 3 } \cline { 5 - 6 } dendrimer & \multicolumn{1}{c}{ water } & mTeSR & & Water & mTeSR \\
G1 & $1.96 \pm 0.32$ & $3.14 \pm 0.33$ & & $7.08 \pm 0.68$ & $-2.62 \pm 2.04$ \\
G5 & $7.90 \pm 0.98$ & $4.82 \pm 1.26$ & & $12.64 \pm 2.95$ & $0.84 \pm 1.30$ \\
G7 & $15.34 \pm 2.30$ & $5.26 \pm 1.40$ & & $20.07 \pm 1.80$ & $7.51 \pm 0.63$ \\
G4.5COOH & $2.90 \pm 0.64$ & $5.52 \pm 1.25$ & & $-0.61 \pm 1.32$ & $-4.41 \pm 0.12$ \\
G5OH & $11.24 \pm 2.00$ & $5.56 \pm 1.19$ & & $-0.09 \pm 0.68$ & $-8.65 \pm 0.52$ \\
\hline
\end{tabular}

Table 2

\begin{tabular}{llll} 
& \multicolumn{1}{c}{ forward } & \multicolumn{1}{c}{ reverse } & \multicolumn{1}{c}{$T_{\mathrm{m}}$} \\
Nanog & AGCCTCTACTCTTCCTACCACC & TCCAAAGCAGCCTCCAAGTC & 60 \\
Nestin & CAGCGTTGGAACAGAGGTTGG & TGGCACAGGTGTCTCAAGGGTAG & 58 \\
Gata6 & CCATGACTCCAACTTCCACC & ACGG AGGACGTGACTTCGGC & 52 \\
Oct4 & GACAACAATGAAAATCTTCAGGAGA & TTCTGGCGCCGGTTACAGAACCA & 60 \\
Tubulin & AACAGCACGGCCATCCAGG & CTTGGGGCCCTGGGCCTCCGA & 58 \\
Foxa2 & ACGACTGTTTCCTGAAGGT & TTGAAGGCGTAGTGGTGT & 52 \\
GAPDH & ACCACAGTCCATGCCATCA & TCCACCACCCTGTTGCTGTA & 60 \\
\hline
\end{tabular}

(Thermo Scientific, Waltham, MA) to remove the unconjugated FITC, according to the manufacturer's instructions. Purified FITC-G5 conjugate was subsequently lyophilized and stored at $4{ }^{\circ} \mathrm{C}$ until use. hESCs grown on a matrigel-coated thin glass dish for 3 days were incubated in Hoechst 33342 (Enzo, NY) diluted 1:1000 in PBS for 10 min and washed with PBS thrice. FITC-G5 dissolved in $\mathrm{mTeSR}$ at a concentration of $10 \mu \mathrm{M}$ was added to the hESCs, at which the time point was set at zero. Endocytosis of Hoechst 33342 and FITC-G5 by hESCs was imaged using confocal microscopy with three-dimensional sectioning for nine layers in $1.78 \mu \mathrm{m}$ per layer and every $9 \mathrm{~min}$ for a total of $180 \mathrm{~min}$. Fluorescent images and average fluorescence intensity per cell at different time points were presented in nine layers.

Reverse Transcriptase-Polymerase Chain Reaction. hESCs seeded in a 24-well plate at a density similar to the cytotoxicity study were allowed to attach overnight. They were incubated with fresh daily exchanged medium containing dendrimers of $1,10,50$, or $100 \mu \mathrm{M}$ for 3 days. RNA was extracted from dendrimer-treated hESCs using RNA extraction kit (TIANGEN, Beijing, China) according to the manufacturer's instructions with an in-column DNase digestion step. cDNA was synthesized using ReverTra Ace-a (TOYOBO, Osaka, Japan) following the manufacturer's instructions with $1 \mu \mathrm{g}$ of RNA per reaction in a total volume of $20 \mu \mathrm{L}$. A reverse transcriptase-polymerase chain reaction was carried out using KOD SYBR qPCR Mix (TOYOBO, Osaka, Japan) following the manufacturer's instructions. Relative gene expression to control was presented. Different annealing temperatures and primer pairs used were as follows (see Table 2).

Immunocytochemistry Assay. Dentrimer-treated hESCs, as operated in reverse transcriptase-polymerase chain reaction, were fixed with $4 \%$ PFA for $15 \mathrm{~min}$ at room temperature. After a PBS wash for 5 min thrice, the cells were permeabilized using $0.4 \%$ Triton in PBS (as required for the antibody) for $10 \mathrm{~min}$ and blocked in a buffer consisting of $1 \% \mathrm{BSA}$ in PBS for $60 \mathrm{~min}$ at $37^{\circ} \mathrm{C}$ before being incubated with primary antibody diluted 1:50-1:300 in blocking buffer overnight at $4{ }^{\circ} \mathrm{C}$. The primary antibodies were as follows: Alexa Fluor 488 conjugated Oct-4A (CST, Boston, MA), Alexa Fluor 647 conjugated Nanog (CST, Boston, MA), and E-cadherin (CST, Boston, MA). The cells were further washed seven times in PBS at room temperature and incubated with secondary antibody (Dylight 594 donkey anti rabbit, Abcam, Cambridge, UK) diluted 1:500 in blocking buffer for $1 \mathrm{~h}$ at $37^{\circ} \mathrm{C}$. After being washed in PBS seven times, the cells were incubated in Hoechst 33342 diluted 1:1000 in PBS for $15 \mathrm{~min}$, washed three times with $\mathrm{PBS}$, and stored at $4{ }^{\circ} \mathrm{C}$ before being observed using confocal laser scanning microscopy. At least 20 cells were imaged in one experimental group. The representative fluorescence images of Nanog and quantitative measurement of fluorescence intensity were presented.
Statistical Analysis. All experiments were repeated at least three times. Statistical analyses were performed using GraphPad Prism 5.0 for Windows (La Jolla, CA). The results are presented as the mean \pm SEM. Unpaired, Student's $t$-test was performed to determine the statistical significance of differences between any two of the three groups for gene transfection, stemness, and differentiation. Two-way ANOVA with the Holm-Sidak test was used to assess the significant differences for cellular viability.

\section{RESULTS}

Characterization of Dendrimer. The particle size and zeta potential of dendrimers in water and mTeSR medium were tested (Table 1). In water solution, the particle size and zeta potential of amine-ended dendrimers were increased with the generations (G1 vs G5 vs G7). The size of G5OH was between G5 and G7, and the size of G4.5COOH was closer to G1 rather than G5. The zeta potential of $\mathrm{G} 5 \mathrm{OH}$ and $\mathrm{G} 4.5 \mathrm{COOH}$ was both slightly negative. In mTeSR, the size differences of all tested dendrimers were greatly decreased. The zeta potential of G5 and G7 was slightly and significantly positive, respectively. Intriguingly, the zeta potential of G1 was changed to slightly negative, possibly due to the conjugation of the counterion. The zeta potential of $\mathrm{G5OH}$ and $\mathrm{G} 4.5 \mathrm{COOH}$ was changed to significantly negative.

Gene Transfection in hESCs Using Dendrimer. To study the gene transfection efficiency of dendrimers in hESCs, a fluorescent visible gene expressing EGFP was used. We found that $1-100 \mu \mathrm{M}$ G4.5COOH and $\mathrm{G} 5 \mathrm{OH}$ had no gene transfection capability, mainly due to their highly negative charges in mTeSR. In contrast, $1000 \mu \mathrm{M} \mathrm{G1}, 1$, and 10 and $50 \mu \mathrm{M}$ G5 as well as 0.5 and $1 \mu \mathrm{M}$ G7 showed good transfection efficacies for hESCs (fluorescence images in Figure 1A), which were enhanced 5.5-, 6.2-, 15.9-, 9.0-, 5.7-, and 5.1-fold, respectively, in comparison with that from Lipofectamine 2000. These results were also confirmed by the number of GFP-positive cells (Figure 1B) and corresponding fluorescence intensity quantification in one cell (Figure 1C). We also found that all cells received successful gene transfection grew normally following gene transfection (DIC images in Figure 1A). These results disclosed that EGFP transfection in hESCs was mostly affected by the terminal group (zeta potential), followed by concentration and size of the dendrimers used. 
A
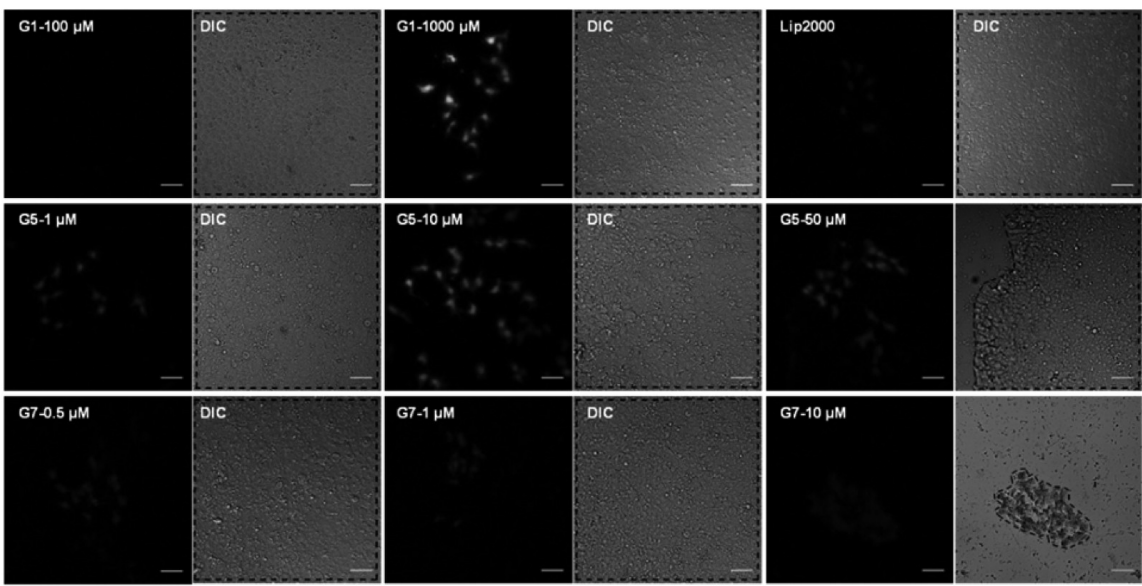

B
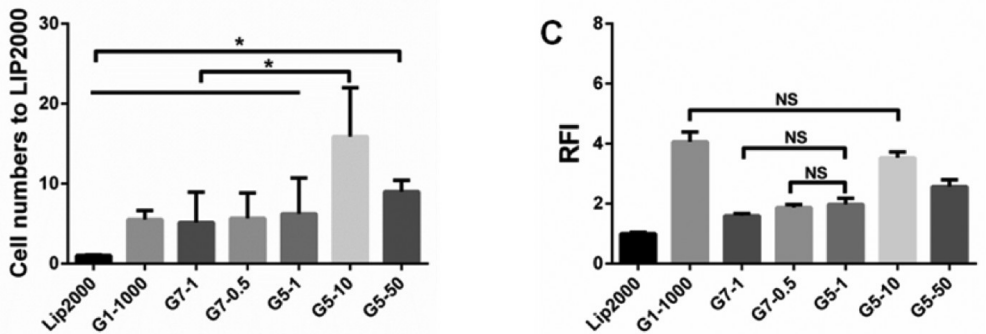

Figure 1. Enhanced GFP gene transfection in hESC H1 colonies using various concentrations of PAMAM dendrimers as vector. (A) Cellular morphology under fluorescence and optical microscope. Note that the absence of cellular morphology at specific dendrimer concentrations was due to the disappearance of green fluorescence signal. (B) Positive green cells relative to Lip $2000(=1)$, ${ }^{*} p<0.05$. (C) Average green fluorescence intensity per

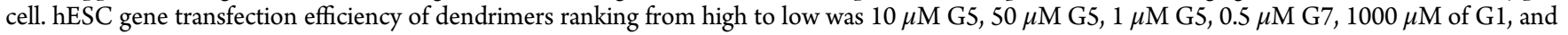
$1 \mu \mathrm{M}$ G7. NS represented no significant difference at $p<0.05$. There were significant differences between any other two groups.

Dendrimer-Induced Cytotoxicity in hESCs. To further uncover the factors affecting the gene transfection efficiency of dendrimers in hESCs, the dendrimer-mediated cytotoxicity in hESCs was assessed using the CCK8 kit. We found that all dendrimers in a concentration range from 1 to $100 \mu \mathrm{M}$ inhibited the growth of hESCs compared with the control (Figure 2). Specifically, the cytotoxicity observed after being exposed with $1 \mu \mathrm{M} \mathrm{G1}, \mathrm{G} 5, \mathrm{G} 4.5 \mathrm{COOH}$, and $\mathrm{G} 5 \mathrm{OH}$ dendrimers was not significantly different. However, when the dendrimer concentration was increased to $10 \mu \mathrm{M}$ or higher, the cellular viability of G5-treated cells decreased to zero, whereas cells with G1, $\mathrm{G} 4.5 \mathrm{COOH}$, or $\mathrm{G} 5 \mathrm{OH}$ dendrimers maintained viability greater than $57 \%$, compared with control (Figure $2 \mathrm{~B}$ ). One to $100 \mu \mathrm{M}$ G7 dendrimer exhibited 100\% of cytotoxicity (Figure 2B), even though $0.5 \mu \mathrm{M}$ G7 had no significant toxicity in hESCs, except for 4-day culture (Figure 2C). Compared with control, G1, and $\mathrm{G} 5 \mathrm{OH}$, the cellular viability of $\mathrm{G} 4.5 \mathrm{COOH}$ dropped sharply with the increase of concentration (Figure $2 \mathrm{~B}$ ), which was also confirmed from the increased dead cells in optical images (Figure 2A). During the 4-day culture period, the viabilities of cells exposed with $1-100 \mu \mathrm{M} \mathrm{G1}, \mathrm{G} 4.5 \mathrm{COOH}$, or $\mathrm{G} 5 \mathrm{OH}$ or $1 \mu \mathrm{M}$ G5 retained a slight increase compared with the viability of each culture at the first day (Figure 2C), suggesting that the dendrimer-caused cytotoxicity was transient. In all cultures, the cytotoxicity of five dendrimers ranking from low to high was from G5OH, G1, G4.5COOH, G5, to G7 (Figure 2), showing that hESC cytotoxicity of dendrimers depends on their terminal group, size, and concentration.

Dynamic Endocytosis of Dendrimer in hESCs. To study the time course of dendrimer access in hESCs and uncover the factors affecting the gene transfection efficiency of dendrimers, the dynamic endocytosis of dendrimers in hESCs was observed using FITC-labeled G5 (FITC-G5) upon its highest transfection efficiency in all dendrimers. In three-dimensional reconstructed images for a total of $180 \mathrm{~min}$ we found that nuclear green intensity gradually increased over time. FITC-G5's red fluorescence appeared on the bottom and surrounding cells, indicating nonspecific adsorption of FITC-G5 on the substrate and distribution of FITC-G5 around cells. The yellow fluorescence, derived from the overlap of nuclear green signal with the red signal from FITC-G5, emerged and gradually increased in hESCs at $72 \mathrm{~min}$ and thereafter, demonstrating the entrance of FITC-G5 into the nucleus (Figure 3A).

Average time-lapsed intensity curves of FITC-G5 and nuclear dye and the ratio of FITC-G5 to nuclear dye per cell in the $\mathrm{z0}-\mathrm{z} 8$ layers (from the closest to farthest away from the substrate) demonstrated endocytosis (leading to an increased intensity), more exocytosis than endocytosis (leading to a decreased intensity), and a balance between endocytosis and exocytosis (leading to a constant intensity) of a fluorescent dye in hESCs. We found the fluorescence intensities of the $\mathrm{z} 0-\mathrm{z} 4$ layers were similar for FITC-G5 and nuclear dye. They were gradually increased and peaked at $45 \mathrm{~min}$. The fluorescence intensities were then gradually decreased to a low level at $72 \mathrm{~min}$ and maintained thereafter. These results disclosed that in the $\mathrm{z} 0-\mathrm{z} 4$ layers the hESCs exhibited more endocytosis than exocytosis in $45 \mathrm{~min}$, more exocytosis than endocytosis between 45 and $72 \mathrm{~min}$, and a balance between exocytosis and endocytosis thereafter. The fluorescence intensities of the $\mathrm{z} 5-\mathrm{z} 8$ layers were slightly different for FITC-G5 and nuclear dye. Within $50 \mathrm{~min}$, the intensity of FITC-G5 maintained a slow increase, while the intensity of nuclear presented initially a high level and slightly 
A

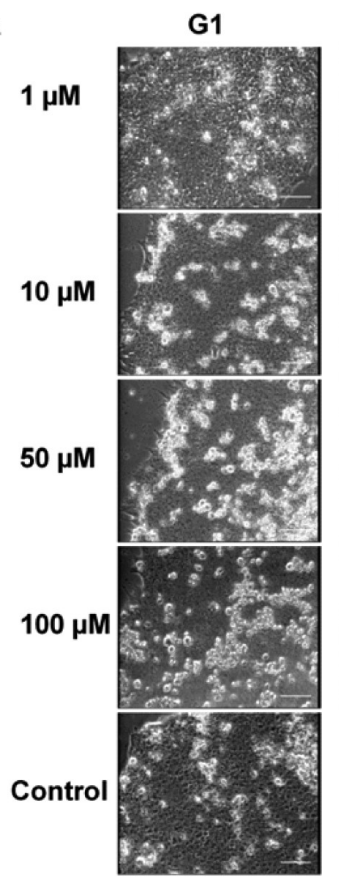

G5

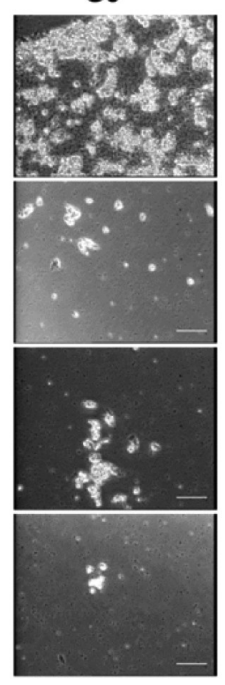

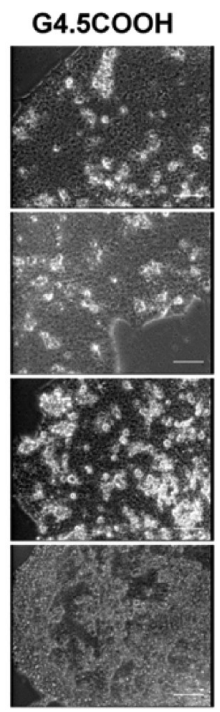

G7

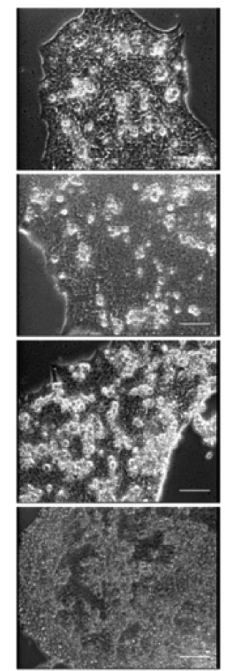

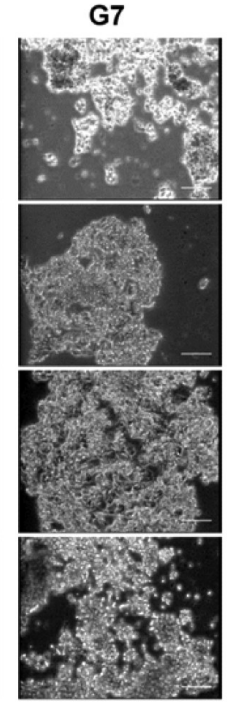

B
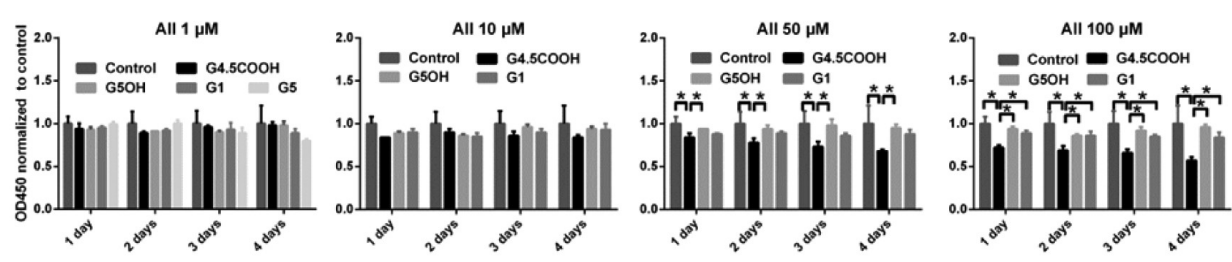

C
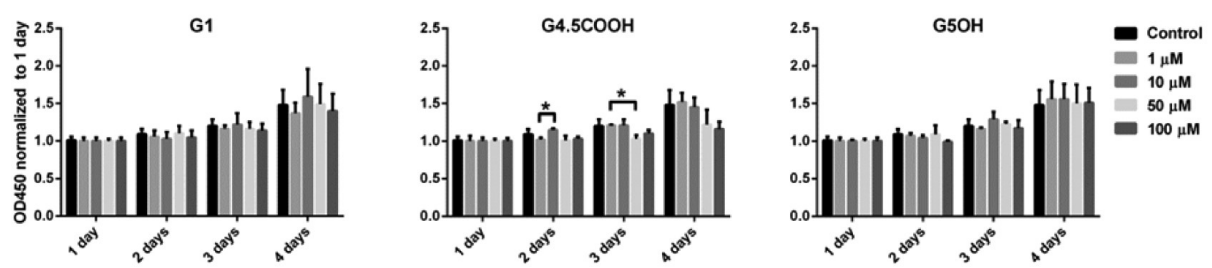

G5
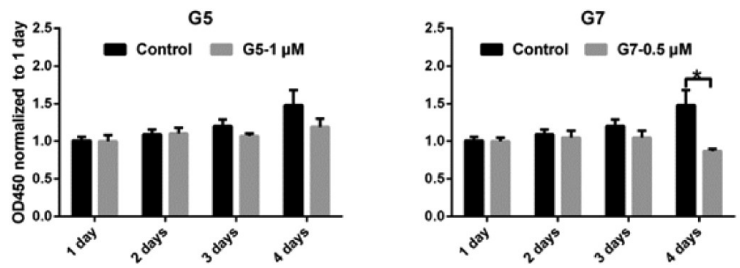

Figure 2. Cytotoxicity of various concentrations of dendrimers on hESC H1 colonies. (A) Cellular morphology at the third day. (B) Plotted as the same concentration of various dendrimers against time. Data are presented as the relative absorbance $(\mathrm{OD})$ at $450 \mathrm{~nm}($ the control $=1)$. (C) Plotted as the same dendrimer of various concentrations against time. Data are presented as the relative $\mathrm{OD}$ at $450 \mathrm{~nm}$ (the first day $=1)$. Cell viability was measured by CCK-8 assay. hESC cytotoxicity of dendrimers ranking from low to high was G5OH, G1, G4.5COOH, G5, and G7.

decreased to a low peak. Both fluorescence intensities of FITC-G5 and nuclear dye were then sharply increased to a high level at $72 \mathrm{~min}$ and maintained thereafter. These results revealed that, in the $\mathrm{z} 5-\mathrm{z} 8$ layers, hESCs exhibited slightly more endocytosis than exocytosis for FITC-G5 but more exocytosis than endocytosis for nuclear dye within $50 \mathrm{~min}$. At the duration between 50 and $72 \mathrm{~min}$, endocytosis was more than exocytosis for both dyes. A balance between exocytosis and endocytosis was achieved thereafter (Figure 3B).

Dendrimer-Induced Stemness or Differentiation of hESCs. To assess the effect of dendrimers on the pluripotency and differentiation of hESCs, the reverse transcriptase-polymerase chain reaction was performed to uncover the RNA expression of pluripotent (Nanog, Oct4), endodermal (Foxa2, Gata6), and ectodermal (Nestin, Tubulin) markers. No significant difference was found among the cells tested (Figure 4).

To test the influence of dendrimers on the maintenance of pluripotency, three pluripotent markers in hESCs, including Oct-4, Nanog, and E-cadherin, were analyzed using immunofluorescence assays. Oct-4 is a protein critically involved in the self-renewal of undifferentiated embryonic stem cells and is frequently used as a marker for undifferentiated cells. Too much or too little Oct-4 causes differentiation of hESCs. ${ }^{22}$ Nanog is a transcription factor in ESCs and is thought to be a key factor in 


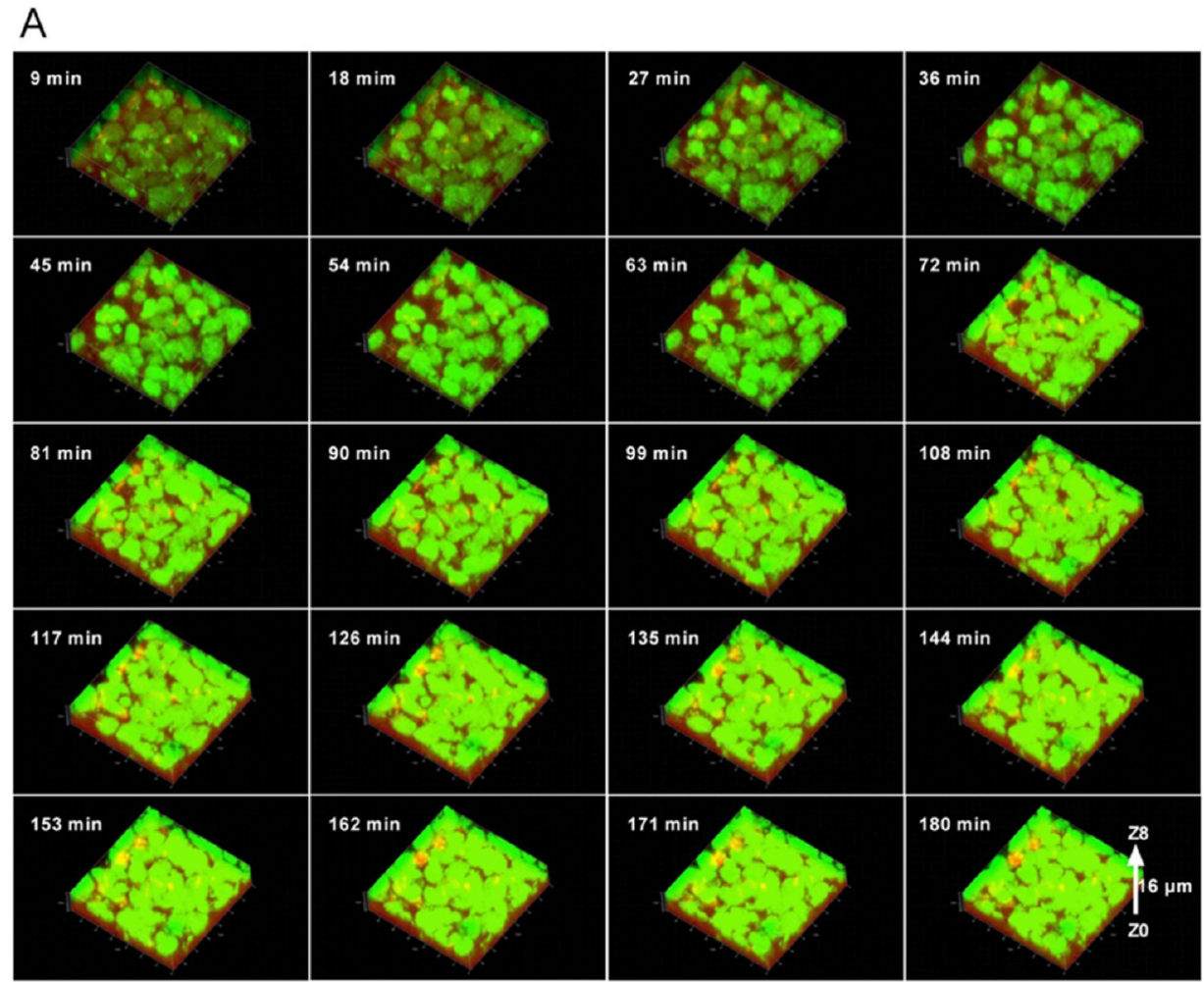

B
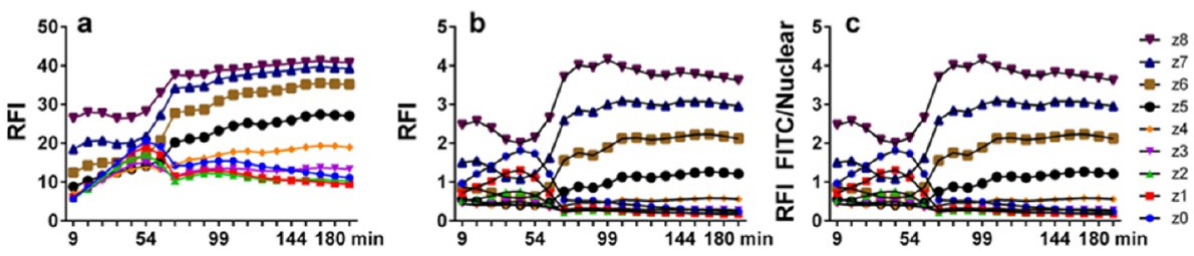

Figure 3. Endocytosis of FITC-labeled G5 (FITC-G5) dendrimer in hESC H1 colonies. (A) Time-lapsed three-dimensional images. Green and red fluorescence signals represented the stained nucleus and FITC-G5, respectively. (B) Average time-lapsed intensity curves of FITC-G5 (a), nuclear dye (b), and the ratio of FITC-G5 to nuclear dye per cell (c). Hoechst 33342 for nuclear staining was added 10 min prior to the time (=0) FITC-G5 was added. The $\mathrm{z} 0-\mathrm{z} 8$ layers represented the closest to farthest layers relative to the substrate at a layer height of $1.78 \mu \mathrm{m}$. Dendrimer endocytosis was confirmed, but their exocytosis was prominent after $50 \mathrm{~min}$ in hESCs.
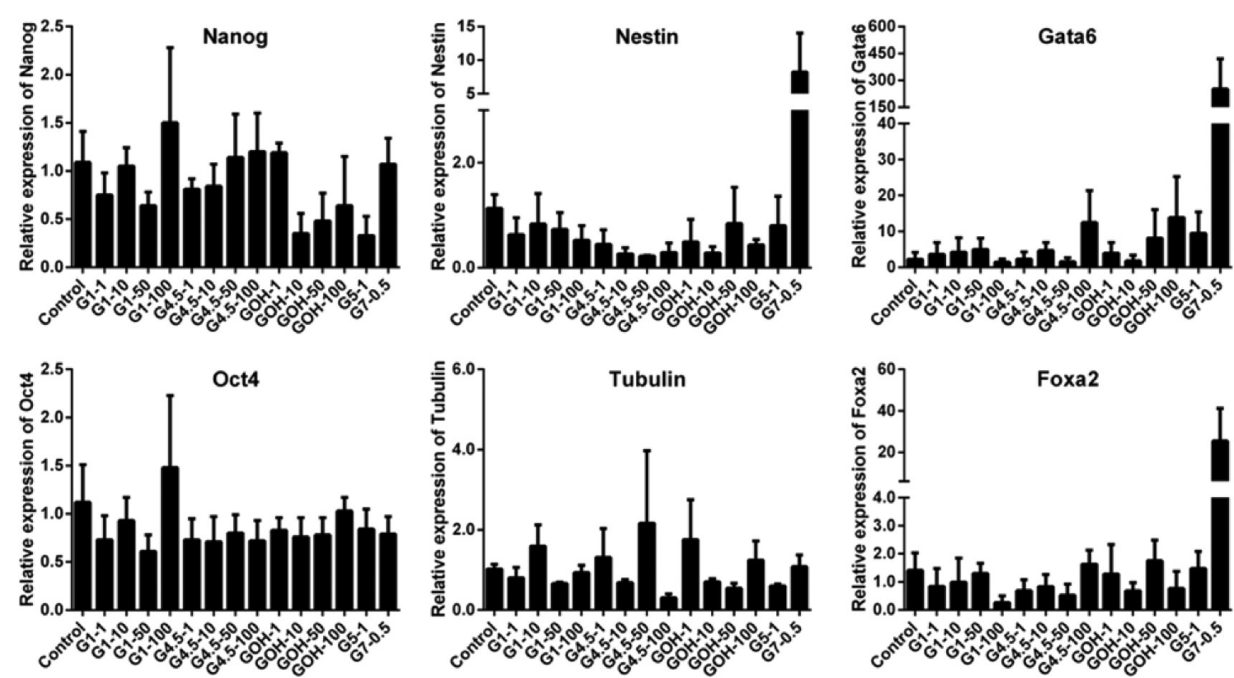

Figure 4. Relative gene expressions (the control $=1$ ) at the third day for pluripotency genes Nanog and Oct-4, ectodermal genes Nestin and Tubulin, and endoderm genes Gata 6 and Foxa 2 of hESC H1 colonies treated with various concentrations of dendrimers, determined by qPCR assay. No significant difference was found among the cells tested. 
maintaining pluripotency and functions in concert with other factors such as Oct4 to establish ESC identity. ${ }^{23}$ E-cadherin is a single-pass transmembrane glycoprotein that regulates localization of cell surface molecules and enhances colony formation and self-renewal in hESCs. ${ }^{24}$ Nanog images were presented as an example marker (Figure 5). Quantitative analyses showed that,

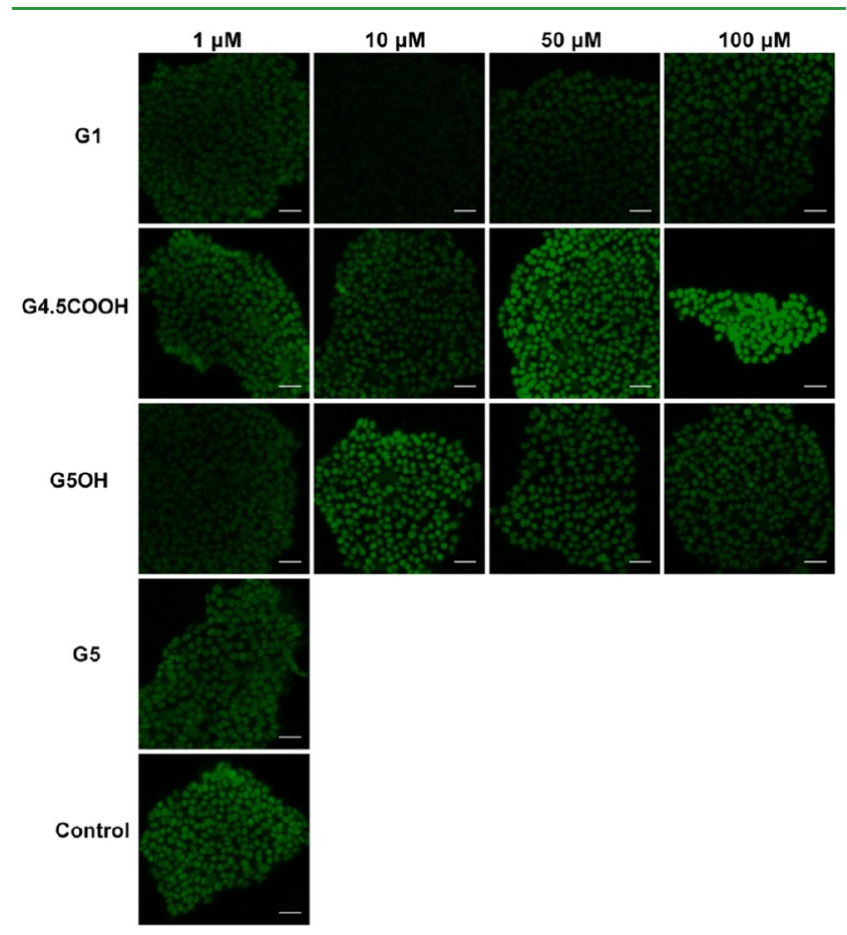

Figure 5. Immunofluoresence images of Nanog at the third day for hESC H1 colonies treated with various concentrations of dendrimers. Note that the absence of images at 10,50 , or $100 \mu \mathrm{M}$ for G5 was due to the disappearance of green fluorescence signal for its high cytotoxicity to the cells.

compared with control, transfection with 1-100 $\mu \mathrm{M} \mathrm{G1}$, $\mathrm{G} 4.5 \mathrm{COOH}$, or $\mathrm{G} 5 \mathrm{OH}$ as well as $1 \mu \mathrm{M}$ G5 maintained or increased hESC expression of Oct-4. From 1 to $100 \mu \mathrm{M} \mathrm{G} 1$ and $1 \mu \mathrm{M}$ G5 maintained or decreased Nanog expression, and $1-100 \mu \mathrm{M}$ G4.5COOH maintained or increased Nanog expression. From 1 to $100 \mu \mathrm{M}$ G5OH did not affect Nanog expression. Transfection with $1-100 \mu \mathrm{M} \mathrm{G1}$ and $1 \mu \mathrm{M}$ G5 maintained or decreased the expression of E-cadherin, whereas $1-100 \mu \mathrm{M}$ G4.5COOH maintained or increased expression of E-cadherin. From 1 to $100 \mu \mathrm{M}$ G5OH did not affect E-cadherin expression (Figure 6). Expression levels of three markers in cells transfected with $10-100 \mu \mathrm{M}$ G5 or $1-100 \mu \mathrm{M}$ G7 were too low to be tested, mainly due to the high cytotoxicity of dendrimers. Therefore, only $1-100 \mu \mathrm{M}$ G4.5COOH and G5OH maintained or even profited the expression of three pluripotency markers, in which the same concentration of $\mathrm{G} 4.5 \mathrm{COOH}$ was better than that of G5OH. In all, the ability of a dendrimer to maintain hESC pluripotency ranking from high to low was from $\mathrm{G} 4.5 \mathrm{COOH}$, $\mathrm{G} 5 \mathrm{OH}$, to G1. These results confirmed that the expression of biomarkers in dendrimer-transfected $\mathrm{H} 1$ cells depends on the terminal group followed by the size and concentration of the dendrimers.

\section{DISCUSSION}

Although it is widely tested for how the type, size, and zeta potential of vector affect gene transfection efficiency in cells, ${ }^{25}$ the systematic study in hESCs remains scarce. The goal of this study is to map the characteristics of distinct nanoparticles in transfection efficacy of hESCs. We chose the widely used dendrimer as a model to test the influence of the size and surface group as well as cytotoxicity and endocytosis on the hESC gene transfection. Our work is irrespective of those reports on dendrimer variable gene transfer efficacy in different cell types $^{18,20}$ and different from those observations of mere cytotoxicity but no influence on cellular differentiation of amino functionalized dendrimers for mesenchymal stem cells. ${ }^{26}$ From our data, a parameter set of size, concentration, and zeta potential (end-group identity and position) optimized as $5 \mathrm{~nm}, 10 \mu \mathrm{M}$, and slightly positive charge provides a high transfection efficacy, low cytotoxicity, and well-maintained stemness. By understanding that the optimized parameter set varies with hESC lineage or stem cell types, these results afford insight into improving the gene transfection of stem cells.

Our gene transfection in hESCs showed that the efficiency is mostly affected by the terminal group (zeta potential), followed by concentration and size of the dendrimers used (Figure 1). Only amine-, but not hydroxyl- or carboxyl-, terminated dendrimers have the gene transfection capability, even greater than that of Lipofectamine 2000 in hESCs. This may result from the significant negative charge of hydroxyl- or carboxyl-terminated dendrimers in cell culture media. Ten micromolar G5 transfected the most genes/per cell in the greatest amount of hESCs, much better than G7 and in the range of the previous study about structure/function relationships of PAMAM dendrimers/DNA as gene delivery vehicles. It is indicated that G4 and G7 have an intermediate relative affinity for DNA and yield the prominent efficiency among G2, G4, G7, and G9. ${ }^{27}$ Inconsistent with the previous observation that G7 is significantly more active than G5 since it could form a bigger size of small transient pores in cell membrane than G5 when interacting with cellular membrane (see review in ref 19), we found only a low concentration of G7 ( 0.5 and $1 \mu \mathrm{M})$ received gene transfection in hESCs for its high cytotoxicity. ${ }^{27}$ A high concentration of G1 $(1000 \mu \mathrm{M})$ delivered a similar amount of genes/per cell in less hESCs compared with $10 \mu \mathrm{M}$ G5, promoting the great influences of size and density of amine groups (zeta potential) on gene transfection. To improve the gene transfection of PAMAM dendrimers, many works have been performed. Modification is an effective way. For example, to utilize the targeting capabilities of RGD integrin, RGD motif is conjugated to PAMAM dendrimer G5 and G6, in which the gene delivery is improved 3 -fold in comparison to native dendrimers. ${ }^{28}$ To apply the capacity of lipids to interact with biological membranes, hydrophobic chains are added in G5 PAMAM as corona. Although the zeta potential is decreased and the size is increased, the modified dendrimers exhibited an improved capacity (as high as 4.5 -fold) with similar cytotoxicity, especially when the vectors contain the smallest hydrophobic chains (mean number of $-\mathrm{CH}_{3}$ at 12.6 and mean number of $-\mathrm{CH} 2-$ at 72.3 in one dendrimer molecule) ${ }^{20}$ Change in gene transfection method is also operative. Gene transfection efficiency is enhanced for up to 4-fold by activating dendrimers using heating at $40^{\circ} \mathrm{C}$ for 8 or $48 \mathrm{~h}^{29}$ or by a novel microfluidic platform. ${ }^{30}$ Our data supported that parameter optimization is preferential for transfection of dendrimers into hESCs, most likely on their end-group identity and size.

We found that hESC cytotoxicity of dendrimers ranking from low to high was from G5OH, G1, G4.5COOH, G5, to G7 (Figure 2). Previous observations disclosed that the cytotoxicity of PAMAM dendrimers increases with generation, independent 

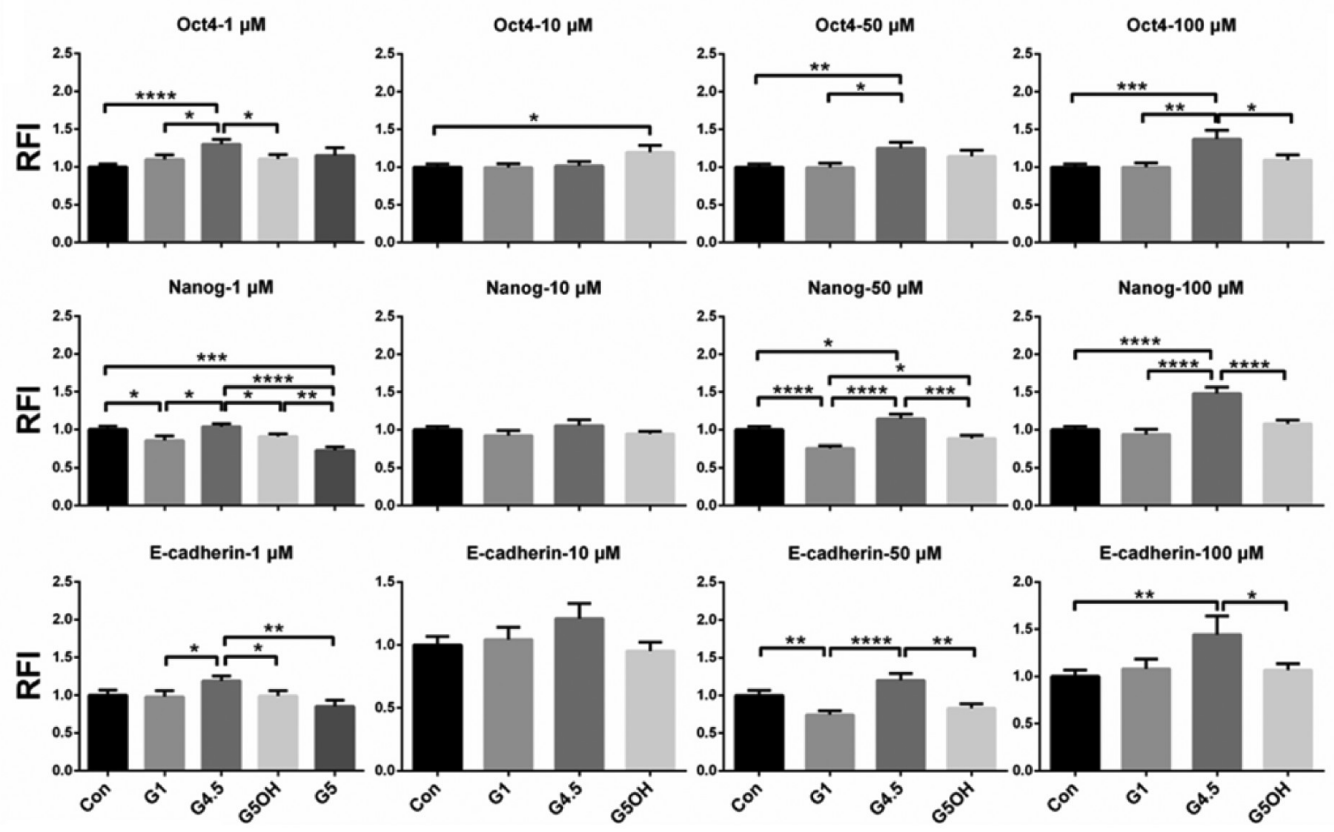

Figure 6. Quantitative analyses of relative fluorescence intensity (RFI, the control = 1) for Oct-4, Nanog, and E-cadherin at the third day for hESC H1 colonies treated with various concentrations of dendrimers. The ability of a dendrimer to maintain hESC pluripotency ranking from high to low was from G4.5COOH, G5OH, to G1.

of surface charge, and that the anionic dendrimers of carboxylate surface are noncytotoxic over a broad concentration range (see review in ref 19). We also revealed the low cytotoxicity of carboxyl-ended dendrimer in hESCs. Furthermore, we disclosed the relatively lower toxicity of hydroxy-ended dendrimer in comparison with the similar size of amine- and carboxyl-ended dendrimers in hESCs in which the low toxicity of carboxyl- and hydroxy-ended dendrimers may result from their significant negative zeta potential. In addition, great impact of zeta potential on hESC toxicity was revealed when comparing the cytotoxicity of G5 and G7, where G7 was more severe than G5. G5 and G7 have similar particle size (4.82 vs $5.26 \mathrm{~nm}$ ) in mTeSR medium but big variance in zeta potential ( $0.84 \mathrm{vs} 7.51 \mathrm{mV}$ ) (Table 1$)$. To reduce the cytotoxicity of amine-ended PAMAM dendrimer, changing the primary amines to secondary or tertiary amines or quaternization and shielding of surface groups, e.g., through covalent attachment of C12 lauroyl groups or PEG 2000, were found to be effective. ${ }^{19}$

Our results confirmed the endocytosis and significant exocytosis of dendrimer into and from hESCs (Figure 3 ) and also showed that the ability of a dendrimer to maintain hESC pluripotency ranking from high to low was from $\mathrm{G} 4.5 \mathrm{COOH}$, $\mathrm{G5OH}$, to G1 (Figures 4 and 6). Combining the chemical structure of dendrimer, the previous modifications on PAMAM dendrimer and our results of gene transfection, cytotoxicity, endocytosis, and stemness/differentiation, the overall size and zeta potential of dendrimers should be considered in the design of hESC gene transfection vector. A size of around $5 \mathrm{~nm}$ and a slightly positive zeta potential may be feasible. In more detail, positively charged primary, secondary, or tertiary amines are needed to compact genes, which may be arranged in the relative inner layer to shield their cytotoxicity. By contrast, the hydroxyl or carboxyl groups can be arranged in the outer layer to directly contact with hESCs to exhibit low toxicity and good pluripotent maintenance. In addition, lowering the exocytosis from hESCs and increasing the binding of dendrimer/genes with
hESC nucleus are effective ways to improve gene transfection efficiency.

\section{CONCLUSION}

In summary, we demonstrated that amine-, but not hydroxyl- or carboxyl-, terminated dendrimers have a gene transfection capability greater than that from Lipofectamine 2000 in hESCs, where $10 \mu \mathrm{M}$ G5 has the greatest efficiency. These types of dendrimers could be detrimental to the growth and pluripotent maintenance of hESCs. Dendrimer exocytosis in hESCs is prominent after $50 \mathrm{~min}$. Careful consideration of size, concentration, and zeta potential, particularly the identity and position of groups, as well as minimized exocytosis should be taken into account in the design of cationic nanoparticles used for hESC gene delivery.

\section{AUTHOR INFORMATION}

\section{Corresponding Authors}

*E-mail: panj@cqu.edu.cn.

*E-mail: mlong@imech.ac.cn.

\section{Author Contributions}

The manuscript was written through contributions of all authors. All authors have given approval to the final version of the manuscript.

\section{Author Contributions}

${ }^{\S}$ C.L. and D.L. contributed equally to this work.

\section{Notes}

The authors declare no competing financial interest.

\section{ACKNOWLEDGMENTS}

We thank Dr. Ye-Guang Chen from Tsinghua University for providing us $\mathrm{H} 1 \mathrm{hES}$ cells upon Simple Letter Agreement between Dr. Chen's and Dr. Long's laboratories that is approved by WiCell Research Institute. This work was supported by National Natural Science Foundation of China grants 31110103918 and 31470907, Chinese Academy of Sciences 
Strategic Priority Research Program grants XDA01030604 and XDA04020219, National High Technology Research and Development Program of China grant 2011AA020109, and Fundamental Research Funds for the Central Universities of China grant CQKLBST-2012-002.

\section{REFERENCES}

(1) Rushing, S. N.; Herren, A. W.; Lieu, D. K.; Li, R. A. Manipulations of microRNA in Human Pluripotent Stem Cells and Their Derivatives. Methods Mol. Biol. 2011, 690, 107-120.

(2) Hoelting, L.; Scheinhardt, B.; Bondarenko, O.; Schildknecht, S.; Kapitza, M.; Tanavde, V.; Tan, B.; Lee, Q. Y.; Mecking, S.; Leist, M.; Kadereit, S. A 3-dimensional Human Embryonic Stem Cell (hESC)derived Model to Detect Developmental Neurotoxicity of Nanoparticles. Arch. Toxicol. 2013, 87 (4), 721-733.

(3) Kim, Y. S.; Hwang, K. A.; Go, R. E.; Kim, C. W.; Choi, K. C. Gene Therapy Strategies Using Egineered Stem Cells for Treating Gynecologic and Breast Cancer Patients (Review). Oncol. Rep. 2015, 33 (5), 2107-2112.

(4) Loh, X. J.; Wu, Y. L. Cationic Star Copolymers Based on Betacyclodextrins for Efficient Gene Delivery to Mouse Embryonic Stem Cell Colonies. Chem. Commun. 2015, 51 (54), 10815-10818.

(5) Cao, F.; Xie, X.; Gollan, T.; Zhao, L.; Narsinh, K.; Lee, R. J.; Wu, J. C. Comparison of Gene-transfer Efficiency in Human Embryonic Stem Cells. Mol. Imaging Biol. 2010, 12 (1), 15-24.

(6) Costa, M.; Dottori, M.; Sourris, K.; Jamshidi, P.; Hatzistavrou, T.; Davis, R.; Azzola, L.; Jackson, S.; Lim, S. M.; Pera, M.; Elefanty, A. G.; Stanley, E. G. A Method for Genetic Modification of Human Embryonic Stem Cells Using Electroporation. Nat. Protoc. 2007, 2 (4), 792-796.

(7) Braam, S. R.; Denning, C.; Van den Brink, S.; Kats, P.; Hochstenbach, R.; Passier, R.; Mummery, C. L. Improved Genetic Manipulation of Human Embryonic Stem Cells. Nat. Methods 2008, 5 (5), 389-392.

(8) Xia, X.; Zhang, Y.; Zieth, C. R.; Zhang, S. C. Transgenes Delivered by Lentiviral Vector are Suppressed in Human Eembryonic Stem Cells in a Promoter-dependent Manner. Stem Cells Dev. 2007, 16 (1), 167176.

(9) Smith-Arica, J. R.; Thomson, A. J.; Ansell, R.; Chiorini, J.; Davidson, B.; McWhir, J. Infection Efficiency of Human and Mouse Embryonic Stem Cells Using Adenoviral and Adeno-associated Viral Vectors. Cloning Stem Cells 2003, 5 (1), 51-62.

(10) Green, J. J.; Zhou, B. Y.; Mitalipova, M. M.; Beard, C.; Langer, R.; Jaenisch, R.; Anderson, D. G. Nanoparticles for Gene Transfer to Human Embryonic Stem Cell Colonies. Nano Lett. 2008, 8 (10), 31263130.

(11) Beloor, J.; Ramakrishna, S.; Nam, K.; Seon Choi, C.; Kim, J.; Kim, S. H.; Cho, H. J.; Shin, H.; Kim, H.; Kim, S. W.; Lee, S. K.; Kumar, P. Effective Gene Delivery into Human Stem Cells with A Cell-targeting Peptide-modified Bioreducible Polymer. Small 2015, 11 (17), 20692079.

(12) Byrne, S. M.; Mali, P.; Church, G. M. Genome Editing in Human Stem Cells. Methods Enzymol. 2014, 546, 119-138.

(13) Liu, W.; Frank, J. A. Detection and Quantification of Magnetically Labeled Cells by Cellular MRI. Eur. J. Radiol. 2009, 70 (2), 258-264.

(14) Shundo, C.; Zhang, H.; Nakanishi, T.; Osaka, T. Cytotoxicity Evaluation of Magnetite (Fe3O4) Nanoparticles in Mouse Embryonic Stem Cells. Colloids Surf., B 2012, 97, 221-225.

(15) Pi, Q. M.; Zhang, W. J.; Zhou, G. D.; Liu, W.; Cao, Y. Degradation or Excretion of Quantum Dots in Mouse Embryonic Stem Cells. BMC Biotechnol. 2010, 10, 36.

(16) Bunting, K. D. ABC Transporters as Phenotypic Markers and Functional Regulators of Stem Cells. Stem Cells 2002, 20 (1), 11-20.

(17) Kievit, F. M.; Wang, F. Y.; Fang, C.; Mok, H.; Wang, K.; Silber, J. R.; Ellenbogen, R. G.; Zhang, M. Doxorubicin Loaded Iron Oxide Nanoparticles Overcome Multidrug Resistance in Cancer in Vitro. J. Controlled Release 2011, 152 (1), 76-83.

(18) Ziraksaz, Z.; Nomani, A.; Soleimani, M.; Bakhshandeh, B.; Arefian, E.; Haririan, I.; Tabbakhian, M. Evaluation of Cationic
Dendrimer and Lipid as Transfection Reagents of Short RNAs for Stem Cell Modification. Int. J. Pharm. 2013, 448 (1), 231-238.

(19) Dufes, C.; Uchegbu, I. F.; Schatzlein, A. G. Dendrimers in Gene Delivery. Adv. Drug Delivery Rev. 2005, 57 (15), 2177-2202.

(20) Santos, J. L.; Oliveira, H.; Pandita, D.; Rodrigues, J.; Pego, A. P.; Granja, P. L.; Tomas, H. Functionalization of Poly(amidoamine) Dendrimers with Hydrophobic Chains for Improved Gene Delivery in Mesenchymal Stem Cells. J. Controlled Release 2010, 144 (1), 55-64.

(21) Jevprasesphant, R.; Penny, J.; Attwood, D.; D’Emanuele, A. Transport of Dendrimer Nanocarriers Through Epithelial Cells via the Transcellular Route. J. Controlled Release 2004, 97 (2), 259-267.

(22) Boyer, L. A.; Lee, T. I.; Cole, M. F.; Johnstone, S. E.; Levine, S. S.; Zucker, J. P.; Guenther, M. G.; Kumar, R. M.; Murray, H. L.; Jenner, R. G.; Gifford, D. K.; Melton, D. A.; Jaenisch, R.; Young, R. A. Core Transcriptional Regulatory Circuitry in Human Embryonic Stem Cells. Cell 2005, 122 (6), 947-956.

(23) Niwa, H.; Miyazaki, J.; Smith, A. G. Quantitative Expression of Oct-3/4 Defines Differentiation, Dedifferentiation or Self-renewal of ES Cells. Nat. Genet. 2000, 24 (4), 372-376.

(24) Soncin, F.; Ward, C. M. The Function of E-cadherin in Stem Cell Pluripotency and Self-renewal. Genes 2011, 2 (1), 229-259.

(25) TurksenK.Preface. Human Embryonic Stem Cell Protocols; Methods in Molecular Biology; Springer: New York, 2016; Vol. 1307.

(26) Goncalves, M.; Castro, R.; Rodrigues, J.; Tomas, H. The Effect of PAMAM Dendrimers on Mesenchymal Stem Cell Viability and Differentiation. Curr. Med. Chem. 2012, 19 (29), 4969-4975.

(27) Braun, C. S.; Vetro, J. A.; Tomalia, D. A.; Koe, G. S.; Koe, J. G.; Middaugh, C. R. Structure/Function Relationships of Polyamidoamine/ DNA Dendrimers as Gene Delivery Vehicles. J. Pharm. Sci. 2005, 94 (2), 423-436.

(28) Pandita, D.; Santos, J. L.; Rodrigues, J.; Pego, A. P.; Granja, P. L.; Tomas, H. Gene Delivery into Mesenchymal Stem Cells: A Biomimetic Approach Using RGD Nanoclusters Based on Poly(amidoamine) Dendrimers. Biomacromolecules 2011, 12 (2), 472-481.

(29) Navarro, G.; Tros de Ilarduya, C. Activated and Non-activated PAMAM Dendrimers for Gene Delivery in Vitro and in Vivo. Nanomedicine 2009, 5 (3), 287-297.

(30) Sharei, A.; Zoldan, J.; Adamo, A.; Sim, W. Y.; Cho, N.; Jackson, E.; Mao, S.; Schneider, S.; Han, M. J.; Lytton-Jean, A.; Basto, P. A.; Jhunjhunwala, S.; Lee, J.; Heller, D. A.; Kang, J. W.; Hartoularos, G. C.; Kim, K. S.; Anderson, D. G.; Langer, R.; Jensen, K. F. A Vector-free Microfluidic Platform for Intracellular Delivery. Proc. Natl. Acad. Sci. U. S. A. 2013, 110 (6), 2082-2087. 